\title{
What BERT Based Language Models Learn in Spoken Transcripts: An Empirical Study
}

\author{
Ayush Kumar, Mukuntha Narayanan Sundararaman and Jithendra Vepa \\ \{ayush, mukunthas, jithendra\}@observe.ai \\ Observe.AI \\ Bangalore, India
}

\begin{abstract}
Language Models (LMs) have been ubiquitously leveraged in various tasks including spoken language understanding (SLU). Spoken language requires careful understanding of speaker interactions, dialog states and speech induced multimodal behaviors to generate a meaningful representation of the conversation. In this work, we propose to dissect SLU into three representative properties: conversational (disfluency, pause, overtalk), channel (speakertype, turn-tasks) and $A S R$ (insertion, deletion, substitution). We probe BERT based language models (BERT, RoBERTa) trained on spoken transcripts to investigate its ability to understand multifarious properties in absence of any speech cues. Empirical results indicate that LM is surprisingly good at capturing conversational properties such as pause prediction and overtalk detection from lexical tokens. On the downsides, the LM scores low on turntasks and ASR errors predictions. Additionally, pre-training the LM on spoken transcripts restrain its linguistic understanding. Finally, we establish the efficacy and transferability of the mentioned properties on two benchmark datasets: Switchboard Dialog Act and Disfluency datasets.
\end{abstract}

\section{Introduction}

Language Models (LM) such as BERT, GPT etc., have led to significant advancements in the field of Natural Language Processing (NLP) by learning representations pre-trained on a vast amount of text data. These LMs have shown to perform well on a multitude of downstream tasks such as question answering, intent, entity and sentiment detection, natural language inference etc. (Devlin et al., 2019). Along with NLP, the proliferation of voice-enabled technologies has resulted in the significance of spoken language understanding (SLU) tasks. The general modus-operandi of SLU systems is to convert voice into text using an
ASR engine and use natural language understanding (NLU) on the transcribed text by modelling conversational and channel properties while being robust to ASR errors. Since spoken conversation boasts of amalgamation of spontaneous speaker interactions, it has become imperative for model architectures to capture multimodal features from text and speech modalities (Georgiou et al., 2019). The aim of these multimodal systems is to capture acoustic information such as pitch, intonation, rate of speech, etc. along with properties such as pause, disfluency, overtalk and turn shifts to synthesize a complete understanding of what is being spoken and how it is being spoken. While some of these properties rely on speech signals and can only be captured by acoustic systems, properties such as pauses, disfluency, overtalk, turn shifts, speech recognition errors etc. get passed onto the lexical domain in the form of spoken transcripts and metainformation. Few works suggest that the auxiliary tasks such as speaker intent detection (Aldeneh et al., 2018), filler prediction (Hara et al., 2018), pause and speaking-rate (Weiner et al., 2017) help to train a robust model for downstream tasks. Thus, there is a need for a vigilant language model capable of encoding the multi-dimensional properties of a SLU dataset to reduce dependence on auxiliary information and/or multimodal inputs. However, in order to come up with such LM for SLU domain, a pre-requisite is to investigate the extent to which an LM already encodes these properties.

In this work, we propose to categorize the spoken language understanding into distinctive properties: 1) Conversational: encompass spontaneous speech properties such as pauses, disfluency, overtalk; 2) Channel: properties that capture speaker turns such as speaker-type, turn-taking; 3) ASR: properties such as error type (insertion, deletion, substitution). While core focus in this work is to not propose a state-of-the-art language model, we believe that unveiling the behavior of LM on these properties 
will not only help us to understand the encoded information in a pre-trained model, but will also influence architectural designs to build upon the findings. Thus, we limit our experiments to utilize only lexical tokens present in the spoken transcripts. Specifically, we aim to answer the following research questions (RQ) in our work:

- RQ1: Can language model encode the distinctive properties of SLU datasets in absence of any speech cues?

- RQ1a: Does LM representations capture conversational properties such as disfluency, overtalk, pause?

- RQ1b: How well can LM encapsulate channel understanding: speaker identity of a channel and turn-tasks?

- RQ1c: Can LM identify insertion, substitution and deletion errors in the spoken transcripts and encode word error rate?

- RQ2: How much of linguistic understanding is retained by a LM trained on noisy spoken transcripts?

- RQ3: Can an LM infused with such properties perform better on an unseen spoken language dataset compared to a vanilla LM?

To answer RQ1, we frame a set of probing tasks (Table 1, Section 2). Probing tasks are a introspection mechanism to unearth the properties encoded in the neural model (LM) (Alain and Bengio, 2017; Tenney et al., 2019). Depending on the task, a probe model can be a classification or a regression setup that is trained on LM-extracted features. The weights of LM are frozen while training the probe model. If the result on a probing task is good enough, we say that the property under the scanner is encoded in the neural model. For RQ2, we evaluate LM on a couple of linguistic properties: dependency relations and entity-value mapping. Finally, we infuse the conversational, channel and ASR properties via a multi-task (MTL) framework trained on probe tasks and evaluate the trained model on unseen spoken language tasks (RQ3). The empirical results and analysis reveal the following insights:

1. LM encodes the conversational properties such as pause and overtalk surprisingly well (RQ1a). Channel properties are captured well enough for only speaker role prediction while
LM finds itself struggling to encode information relevant for turn-tasks $(R Q 1 b)$. ASR properties are poorly captured in the LM. While the model can detect if a token is erroneous, it is inaccurate in predicting the error type. Interestingly, ASR transcript pre-trained LM detects substitution much better than vanilla LM in comparison to insertion and deletion errors $(R Q 1 c)$.

2. Once trained on spoken transcripts, the LM vastly loses its understanding of dependency relations. Model also scores poorly on entityvalue mapping $(R Q 2)$.

3. An LM fine-tuned on probing tasks with a multi-task learning (MTL) framework results in an improved performance on Switchboard Dialog Act and Disfluency corpus. This shows that language model representations which encode such properties perform better on downstream tasks $(R Q 3)$.

\section{Probing Tasks (RQ1)}

In this section, we define probing tasks with respective examples. We also describe the dataset creation methodology in detail in Section A.1.

\subsection{Conversational Properties (RQ1a)}

\subsubsection{Disfluency Detection}

An important characteristic of spontaneous speech that makes it distinguishable from a written text or formal speech is the presence of disfluencies. Disfluency can refer to filler words ( $u m, u h)$, repetitions (this this is just not working), false starts (you were okay that's perfectly fine), discourse markers ( $i$ mean) etc. that are an integral part of natural conversations. Despite disfluencies considered as noise to downstream NLU tasks, they indicate distinguishing behaviors such as low confidence of the speaker (Dinkar et al., 2020) and non-native speaker of the language. Hence, identification of disfluent text in spoken transcripts is of prime importance to capture auxiliary speaker behaviors.

In this work, we create a disfluency dataset that has the instances categorized into fluent vs disfluent classes. A probe classifier is set up on this dataset for disfluency detection.

\subsubsection{Pause Identification}

Pauses and silences are natural elements of human interaction. Any spontaneous conversation would contain participants taking pauses and silences indicating situations such as: holds for retrieving 


\begin{tabular}{|c|c|}
\hline Property Type & Probe Tasks \\
\hline Conversational & $\begin{array}{l}\text { Disfluency Detection: Is the given spoken utterance disfluent? } \\
\text { no so no i i don't can i have instead get a refund } \\
\text { well i don't have a i mean i probably do have a provider there } \\
\text { Pause Identification: Does the speaker take long pauses while speaking? } \\
\text { let's see <silence>i see so we haven't tried to go on the website } \\
\text { okay <silence>so what } i \text { see is that your request is already with us } \\
\text { Overtalk Detection: Are two speakers talking over each other? } \\
\text { i'm [yes] not [i know] referring to [how] the [it works] transaction } \\
\text { i [you] thought [want] that [me to] you [do] gotta [that] do [for you] } \\
\text { Question Classification: Did the speaker ask any question? } \\
\text { [entity]: and your date of birth please } \\
\text { [choice]: so you want to go with monthly plan or yearly }\end{array}$ \\
\hline Channel & $\begin{array}{l}\text { Speaker Role: Who is the speaker for a given utterance? } \\
\text { agent: you can cancel anytime you are not obligated to write to us } \\
\text { customer: thank you i tried yesterday } i \text { was told to call back today } \\
\text { Response Length: Is the expected response to current utterance is short or long? } \\
\text { short: <can i get you full name please }><\text { it's johnson rest }> \\
\text { long: <may i know what didn't work }><\text { when } i \text { came to webpage ... > } \\
\text { Turn Taking: Has speaker completed its turn? } \\
\text { turn-break: <appreciate your reply } i \text { 'll get that done for you }><\text { silence }> \\
\text { turn-continue: }<i \text { get that let me verify that for you }<\text { silence }>\text { okay i've } \\
\text { pushed data from my end you should hear back in } 48 \text { hours }>\end{array}$ \\
\hline ASR Errors & $\begin{array}{l}\text { Error Type: What category of ASR error does a particular token belong to? } \\
\text { reference: <customer resolution is our primary motive> } \\
\text { hypothesis: <customer resolution is hour primary motive> } \\
\text { hour: substitution error } \\
\text { WER Score: What is the word-error-recognition score for the transcript? } \\
\text { reference: <customer resolution is our primary motive> } \\
\text { hypothesis: <customer resolution is hour primary motive> } \\
\text { WER: } 16.67\end{array}$ \\
\hline
\end{tabular}

Table 1: Representative examples for each probing task. Special tokens or markers are pruned before running the probe setup.

information, transition to a new topic of discussion, silence to gather thoughts, etc. Identification of pauses can make the language model understand the current dialog state viz., information exchange, call-hold, turn-shifts, topic-change etc.

In this work, we treat silences longer than $5 \mathrm{sec}$. as a pause and pose pause identification as a binary classification problem with an intent to understand how well an LM encodes the information about the presence or absence pause without taking any speech cues.

\subsubsection{Overtalk Detection}

Overtalk is a phenomenon that occurs when multiple parties are speaking at the same time. While overtalk is inevitable in a natural conversation, too much overtalk in conversations indicate poor listening skills displayed by the involved parties. Detecting overtalk is straightforward in dual-channel (diarized) calls, but in mono-channel (non-diarized) calls the speaker segmentation is not available. Although diarization is a preferred way to identify speakers, such models are non-trivial to train and would contain margin of errors.

We identify overtalks in dual-channel calls and convert the corresponding spoken transcript in a sequence of time-ordered tokens (mono-channel transcript) for the overtalk detection task (Table 1). Overtalk detection is a binary classification task where one class contains text that contains overtalk 
between speakers while the other is a turn uttered by single speaker.

\subsubsection{Question Identification}

An interaction between an agent and a customer hinge around multiple information exchanges often anchored via a question-answer pair. The intent of the question, eg: entity, descriptive, boolean etc. additionally helps to track the anatomy of the call which captures activities such as agent verifying customers (entity questions), agent probing customers to gather complete understanding of the issue (descriptive questions) etc. However, in spoken language, the formal structure of the question is not always followed and often questions are asked in non-surface forms (and your account number) instead of can you provide your account number). Thus, question identification is a challenging task in SLU domain. We frame question identification as a multi-class setup with four classes: entity, descriptive, boolean and choice based.

\subsection{Channel Properties (RQ1b)}

\subsubsection{Speaker Role}

Identity of the current speaker and speaker roles (sender, addressee, observer, etc.) facilitates a better understanding of the dialog state in a conversation (Kim et al., 2020). Speaker roles and identities have also been leveraged in context extraction and response generation in dialog systems (Zhang et al., 2018a). Thus, we formulate a probe task to identify the role of the current speaker in a call. In a call center conversation, there are three speaker roles that co-exist: agent, customer and IVR. We formulate a multiclass classification task to probe the LM on identifying speaker roles.

\subsubsection{Turn Tasks}

Understanding contexts (turns) in a conversation is a key step to frame a better understanding of the conversation. To probe on how well the LM encode the contexts, we posit two turn-tasks:

- Response-Length: We set up a binary classification task where the probe classifier predicts the duration of the next-turn (short or long) given the current and previous turn. This task is an interesting intersection of spoken and written properties of SLU where a turn that is as short as (let me see. oh seems you're correct) in the lexical domain can be as long as a minute in the time domain because of the silence that speaker took while looking-up the information. Hence, we aim to probe LM on not just lexical understanding of tokens but also identify if LM captures such subtleties of spoken transcripts. We consider any turn that lasts up to 30 seconds as a short-response and a turn lasting longer than that as a longresponse.

- Turn-Taking: In a real-time system, the spoken transcript comes in as a batch of tokens. In such a system, it becomes important to understand if the speaker has completed its turn and is ready to listen. Inspired by (Roddy et al., 2018), we formulate the situation as a binary text classification where the task is to predict if the speaker would continue speaking at the end of the utterance or would it handover the turn to the other party. We use the pause markers in the dataset to identify the discourse segments which is used to generate the dataset for turn-taking.

\subsection{ASR Properties (RQ1c)}

\subsubsection{Error Type Prediction}

We formulate this task as a token classification task i.e, a single token of an entire utterance is considered at a time for probing. We define two setups for error type prediction: a) Binary Classifcation: Given a token in spoken transcript, classify if the token is correct or erroneous; b) Multiclass Classification: Given an erroneous token in spoken transcript, classify the error type of the token. In case of deletion, the token next to deleted token is probed for the error type. We believe this is an ideal setup as in real scenario, one can only distinguish a deleted token only when next token is predicted in the ASR transcript.

\subsubsection{Word Error Rate (WER) Prediction}

In downstream tasks such as call summarization, the dialog turns that are highly noisy (contains ASR errors) are undesirable and should not be focused much while generating summaries. To achieve this property, it becomes inevitable for the model to identify such dialog turns with high WERs. Since any such recent summarization system uses language modelling as underlying component, we probe the LM on its ability to predict the WER of a given dialog turn.

\section{Linguistic Understanding of LM (RQ2)}

Deep language models such as BERT have been shown to encode a range of syntactic and semantic 
information with more complex structures represented hierarchically in the higher layers of the model (Conneau et al., 2018; Jawahar et al., 2019). $\mathrm{Li}$ et al. (2020) emphasize on capturing syntactic structure via syntax aware local attention while Sundararaman et al. (2019) propose injecting structural and syntactic information such as parts-ofspeech tags which results in a higher performing machine translation model. Since spoken language transcripts contain spontaneous texts devoid of formal structure and incorrect grammars added with speech to text conversion errors, it becomes imperative to quantify the linguistic structures encoded in the language model. We probe LM for such properties via these setups:

1. Dependency Parsing: Following the work in Clark et al. (2019), we investigate individual attention heads in both directions to probe what aspects of language they have retained after being pre-trained with spoken language transcripts on the benchmark task of dependency parsing. We use Penn Treebank dataset (Marcus et al., 1993) tagged with Stanford's dependencies to report the performance on dependency parsing.

2. Entity-Value Mapping: In an agent-customer conversation, agent usually asks some PII entities to verify the identity of the customer before proceeding further into the call (eg: can you provide me your date of birth and last name). The customer replies with a reference to both entities asked by the agent (eg: sure that would be davis and ninth march ninety four For an entity extraction system to work in such spoken language transcripts, the model should be able to map davis to last name and ninth march ninety four to date of birth. In this work, we pose this problem as a entity-value mapping. Particularly, we compute entity selection accuracy measured by what percent of the time does the head word of a entity value most attend to the head of its entity. For example, if 3 tokens out of ninth march ninety four attends maximum to any of the token in date of birth, we would say that entity selection accuracy is $75 \%$.

\section{Properties Infused LM: A Multi-Task Learning setup (RQ3)}

We hypothesize that an LM that better encodes the conversational, channel and ASR properties in its representation performs better on downstream SLU tasks. In order to verify the hypothesis, we follow a two step pipeline:

1. In the first step, we infuse LM with these properties by fine-tuning both LMs on all probe tasks simultaneously using a multi-task learning (MTL) framework.

2. Next, we freeze the weights of the LMs and evaluate the performance of the probe classifier on a couple of external datasets: Switchboard Dialog Act and Switchboard disfluency for dialog act classification and disfluency detection respectively. It should be noted that none of these datasets are utilized in the pretraining step of the two LMs.

Since MTL-trained LMs get explicit supervision to capture properties needed by probing tasks, their performance on downstream tasks would also indicate the relevance of the proposed probing tasks.

\section{Experimental Setup}

\subsection{Language Model}

We use BERT (Devlin et al., 2019) as our base language model. Since, RoBERTa (Liu et al., 2019), which is a robustly-optimized version of BERT is also trained on masked language modelling task and has been reported to outperform BERT on several NLP tasks, we extend our probe to RoBERTa. We observe a better result, in general, from RoBERTa-base model. Hence, we run further experiments on variations of RoBERTa-base model. Specifically, we compare following setups:

- BERT-base: We use pre-trained BERT-baseuncased model to evaluate out-of-box performance on the probe tasks.

- RoBERTa-base: Since, RoBERTa is a BERTlike model robustly trained with larger dataset, we also utilize pre-trained RoBERTa-base model to report the results.

- Chat-RoBERTa: We pre-train RoBERTa on utterances derived from two dialog corpus: PersonaChat (Zhang et al., 2018b) and MultiOz dataset (Budzianowski et al., 2018). The pretraining is done with MLM task to influence the LM with dialog properties, with an aim to establish a competitive baseline trained on a large spontaneous dialog dataset.

- ASRoBERTa: It is imperative to pre-train LM on large domain data for a better performance. ASRoBERTa is pre-trained on in-domain ASR corpus to evaluate the impact of domain pre- 
training on the performance on probing tasks $\left(\Delta_{\text {domain }}\right)$.

-Oracle: In an ideal setup, it is suggested to finetune the LM on the downstream task for best results. We report results on task fine-tuned model (Oracle) to understand the limits of the LM in an ideal setup compared to in-domain training.

\subsection{Dataset}

In this work, we focus on evaluating the LM on its ability to encapsulate spoken language understanding in English language with a special focus on real-life spontaneous conversations such as call center interactions. Keeping that in mind, we train ASRoBERTa with transcripts derived from two datasets: a) LibriSpeech (Panayotov et al., 2015) as a general-purpose ASR dataset (960 hrs.); b) reallife proprietary dataset ${ }^{1}$ (1000 hrs.). All probing setups are carried out on proprietary datasets. For probing tasks, a total of 10k datapoints are used in the training while validation and test set comprise of $2 \mathrm{k}$ datapoints each. The dataset for each probe task is class-balanced. It is to be noted that we use real life dataset with audio of $8-16 \mathrm{kHz}$. The average word-error-rate (WER) of automatic speech recognition (ASR) system for our dataset is 18.38 . The probe task dataset creation methodology is explained in Section A.1.

To evaluate the efficacy of the probing tasks and study the transferability of LM to unseen data, we also evaluate LM fine-tuned with multi-task learning framework on two external datasets: Switchboard Dialog Act (SWDA) (Calhoun et al., 2010) and Switchboard Disfluency dataset (SWDB Disfluency) (Zayats et al., 2019). SWDA consists of utterances categorized into one of 42 dialog act labels, while SWBD disfluency dataset comes with tokens annotated with disfluency tags. We use the already provided train, test and valid splits of the two datasets to run experiments.

\subsection{Implementation Details}

We use linear probe models (Tenney et al., 2019; Alain and Bengio, 2017) with a cross-entropy loss for classification and mean squared error for regression. The input to probe classifier is the utterance representations derived from $\langle s\rangle$ for all conversational, channel and WER tasks, while we feed contextualized token embeddings for ASR error

\footnotetext{
${ }^{1}$ We cannot release the datasets or trained models due to privacy reasons.
}

type detection in the probing model. In line with probing task evaluation, we accumulate layer wise results for each task. Each setup is trained for a total of 20 epochs and the best model on the validation set is used to report the results on the test set (Table 2).

\section{Results and Analysis}

The results for experiments are presented in Table 2 with the improvements obtained from domain pre-training $\left(\Delta_{\text {domain }}\right)$ and then further with task fine-tuning (Oracle).

On Conversation Properties (RQ1a): Properties including disfluency, pause, overtalk are captured surprisingly well by domain LM (ASRoBERTa) without explicit supervision for these tasks in the pre-training step. An LM aware of pauses, disfluency and overtalk can understand discourse and speaker segmentation better and hence it is an encouraging sign to note decent results from probing classifiers on the conversational tasks. The oracle results, although, show that there is a scope for LM to encode these properties even better. A simple downstream task fine-tuning (Oracle) provides a considerable boost (2-10\%) in F1-score for these tasks.

On Channel Properties (RQ1b): The LM when probed on channel properties performs quite well (83.98\%) on speaker role identification. This shows that ASRoBERTa learns to distinguish speaker roles sufficiently in its pre-training step. Although domain pretraining improves the results by $4.5 \%$, the RoBERTa scores for speaker role identification is close to $80 \%$ indicating that agents and customers play a disntinctive role easily identifiable by the transcript of the speaker itself. On the other side, the results on turn-tasks are below $70 \% \mathrm{~F} 1$ score. One explanation for this is the inherent difficulty of the turn-tasks which requires a capability to anticipate a turn-transition and response generation properties such as response-length determined by the contextual understanding of the speaker turns and dialog state. Oracle experiment improve over by upto 3\% over ASRoBERTa. Relatively lower oracle scores suggest that a robust mechanism is needed to infuse turn-task properties to make LM aware of contextual information.

On ASR Properties (RQ1c): An LM trained on spoken transcripts is able to distinguish tokens that are erroneous from the correct tokens, scoring $73.32 \% \mathrm{~F} 1$ on binary classification probe. How- 


\begin{tabular}{l|l|cc|ccc|c}
\multicolumn{2}{c}{ Probing Task } & $\begin{array}{c}\text { BERT } \\
\text {-base }\end{array}$ & $\begin{array}{c}\text { RoBERTa } \\
\text {-base }\end{array}$ & $\begin{array}{c}\text { Chat } \\
\text {-RoBERTa }\end{array}$ & ASRoBERTa & $\Delta_{\text {domain }}$ & Oracle \\
\hline \multirow{4}{*}{ Conversational } & Disfluency & 64.23 & 65.18 & 70.52 & 73.13 & 2.61 & 74.18 \\
& Pause & 73.67 & 72.45 & 77.31 & 81.29 & 3.98 & 85.20 \\
& Overtalk & 80.09 & 81.46 & 83.75 & 85.15 & 1.40 & 95.45 \\
& Question & 69.42 & 69.94 & 70.98 & 75.41 & 4.43 & 77.87 \\
\hline \multirow{3}{*}{ Channel } & Speaker & 79.18 & 78.45 & 79.39 & 83.98 & 4.59 & 85.90 \\
& Response-Len & 63.28 & 64.30 & 65.50 & 68.11 & 2.61 & 70.55 \\
& Turn-Taking & 65.26 & 65.04 & 66.23 & 67.45 & 1.22 & 70.36 \\
\hline \multirow{5}{*}{ ASR } & Binary $^{*}$ & 67.14 & 68.58 & 72.43 & 73.32 & 0.89 & 75.64 \\
& Insertion $^{\dagger}$ & 55.66 & 56.49 & 58.99 & 60.87 & 1.88 & 61.73 \\
& Deletion $^{\dagger}$ & 60.50 & 58.37 & 62.49 & 64.28 & 1.79 & 66.17 \\
& Substitution $^{\dagger}$ & 48.82 & 49.96 & 50.82 & 55.71 & 4.89 & 54.34 \\
& WER $^{\#}$ & 10.38 & 10.09 & 8.50 & 7.42 & -1.08 & 6.27
\end{tabular}

Table 2: Probing results on all tasks. WER $\#$ is a regression task while others are classification tasks. Tasks with ${ }^{\dagger}$ are class-wise scores of a multiclass setup. Macro-F1 (higher is better) and MAE (lower is better) are reported for classification \& regression respectively. Negative $\Delta$ for WER is a welcome change since lower WER is better.

ever, the model is highly inaccurate in distinguishing the error types. The comparisons between ASRoBERTa and RoBERTa on the three error types show that ASRoBERTa is able to identify substitution errors substantially better $(55.71 \%)$ than RoBERTa $(50.82 \%)$ while has lower gains on insertion and deletion errors $\left(\Delta_{\text {domain }}<2 \%\right)$. Additionally, ASRoBERTa predicts the WER score of an utterance with a mean absolute error of 7.42 which improves by 1.15 WER once trained with downstream task-finetuning. However, Oracle results are only marginally better than ASRoBERTa on insertion and deletion. The substitution scores drops down at the cost of improving the error type detection. Thus, LM representations are insufficient to capture ASR error types and pre-training mechanism needs to be revisited to make model learn these ASR properties effectively.

Meta-Comments on RQ1: Along with the granular analysis on different properties presented above, the macro-analysis shows some interesting insights:

- Default models (BERT-base, RoBERTa-base) perform in close-margins on channel properties, as compared to conversational and ASR properties. We hypothesize that conversational and ASR properties require understanding of spontaneous interactions like pauses, stutters and noisy transcriptions which is not present in the base corpus of BERT-base and RoBERTa-base. On the other hand, channel properties are more generic tasks that require lexical understanding (speaker role) and language generation understanding (turn-tasks) which could still be induced from clean texts.

- A simple pre-training step to induce conversational properties (Chat-RoBERTa) is helpful to realize gains across the tasks. This indicates that we may want to train a better and more suited base-checkpoint for tasks related to dialog systems and spoken language understanding. One of the most simple mechanism would be pre-train a model on publicly available spoken language and/or conversational datasets.

- We observe significant gains in taskfinetuning steps for conversational and channel properties. Hoowever, the absolute results on tasks such as response length and turn-taking may note yet be satisfactory. This calls out a need to identify novel pre-training strategies and/or architecture that could lead to a better base-checkpoint across diverse set of generic tasks related to spoken language understanding.

- Weak results on ASR probes suggest that MLM objective is insufficient for the pretrained language models to effectively distinguish between the erroneous tokens and their error types. It would be interesting to try some ASR-specific language model, such as warped language model (Namazifar et al., 2021) which is trained with two additional training tasks, namely INSERT and DROP where the model is trained to predict where a random token is inserted into or deleted from the input sequence during training.

On Linguistic Understanding (RQ2): The re- 


\begin{tabular}{lccc} 
Relations & Chat-RoBERTa & ASRoBERTa & $\Delta_{r-a s r}$ \\
\hline all & 34.9 & 31.4 & -3.5 \\
\hline \multicolumn{4}{c}{ Most frequent relations } \\
\hline prep & 66.6 & 62.3 & -4.3 \\
pobj & 71.7 & 60.9 & -10.8 \\
det & 87.1 & 78.8 & -8.3 \\
$n n$ & 74.0 & 71.9 & -2.1 \\
nsubj & 56.4 & 54.1 & -2.3 \\
amod & 82.5 & 79.1 & -3.4 \\
dobj & 78.9 & 75.2 & -3.7 \\
advmod & 51.9 & 48.8 & -3.1 \\
aux & 81.4 & 82.1 & 0.7 \\
num & 78.6 & 54.2 & -24.4 \\
\hline \multicolumn{5}{c}{ Relations with highest delta } \\
\hline num & 78.6 & 54.2 & -24.4 \\
ccomp & 54.2 & 35.0 & -19.2 \\
poss & 83.9 & 68.4 & -15.5 \\
conj & 59.0 & 43.8 & -15.2 \\
cc & 54.1 & 41.8 & -12.3
\end{tabular}

Table 3: Results (UAS scores) for attention-based probe on dependency parsing. 10 most frequent dependency relations are reported along with the ones with highest delta $(\Delta)$.

\begin{tabular}{ccc} 
Model & Entity-Value Acc. & ISA\% \\
\hline Chat-RoBERTa & 7.98 & 3.78 \\
+ NSP Task & 38.62 & 41.98 \\
\hline ASRoBERTa & 10.19 & 3.65 \\
+ NSP Task & 42.64 & 46.27
\end{tabular}

Table 4: Entity-Value mapping accuracy with intersentence attention (ISA\%).

sults for attention based probe for dependency parsing is reported in Table 3. For the sake of brevity, we present results for 10 most frequent relations in the dataset. We observe that a language model pre-trained on spoken transcripts (ASRoBERTa) worsens the results obtained by a language model trained on clean text chat (Chat-RoBERTa) by 3.5\% UAS. Additionally, we note that ASRoBERTa performs poor than Chat-RoBERTa in $81.8 \%$ of all dependency relations with num relation having the biggest impact on the performance ( $\Delta=24.4 \%$ ). ASRoBERTa performs marginally better only on a couple of relations (aux: $\Delta=0.7 \%$, prt: $\Delta=$ $0.5 \%$ ). These results conclusively show that ASRoBERTa does not retain its understanding of dependency relations leading to huge drop in many relations' performance. A reasoning for this could be the fact that ASRoBERTa is trained on grammatically noisy and non-punctuated texts. With a number of ASR errors and incoherent statements, the model may have learnt to not pay attention to dependency links.

\begin{tabular}{ccc} 
Model & SWDA & SWBD Disfluency \\
\hline Chat-RoBERTa & 68.71 & 68.39 \\
+ MTL & $\mathbf{6 9 . 7 2}$ & $\mathbf{7 0 . 2 7}$ \\
\hline ASRoBERTa & 67.04 & 72.01 \\
+ MTL & $\mathbf{6 8 . 3 7}$ & $\mathbf{7 5 . 9 5}$
\end{tabular}

Table 5: Accuracy on SWDA and Switchboard Disfluency dataset in a multi-task learning (MTL) setup

In another experiment of entity-value resolution, it is observed that both RoBERTa and ASRoBERTa performs poorly on the task achieving only $7.98 \%$ and $10.19 \%$ respectively on attention-based probing method (Table 4). We hypothesize that this could be a result of model's inability to encode the inter-utterance dependencies in a turn-by-turn dyadic conversation. To validate the hypothesis, we perform these steps:

- We compute inter-sentence attention score as a percentage (ISA\%) of overall attention that includes self-attention, intra/inter-sentence attention and attention to separator tokens. We observe that the average inter-sentence attention is less than 0.1 except for initial two layers. This shows that model does not put enough emphasis on inter-sentence interactions while encoding the given input. The observation is surprising as model was trained with consecutive dialog turns separated by $</ s>$ tokens.

- In order to confirm that poor inter-sentence interaction leads to lower attention and consecutively lower entity-value resolution accuracy, we force the model to learn inter-sentence interactions by training it on a binarized next sentence prediction (NSP) task (Devlin et al., 2019). A model would need to understand the input sequence to correctly predict if the last utterance in the input follows the previous utterance. Once the model is trained on NSP task, we run the attention based probing on entity-value resolution once again and note considerable improvements.

The experiment with entity-value resolution task demonstrates that LM poorly encodes intersentence interactions and thus provides a scope to model these interactions in an effective manner in future works.

On properties infused LM: MTL Setup (RQ3): We show that an LM fine-tuned on all probe tasks in a multi-task learning (MTL) setup performs better than base LM on an unseen corpus (Table 5). MTL improves the accuracy by $1.35 \%$ in SWDA 
task, while it leads to a gain of $3.94 \%$ on the disfluency task for the ASRoBERTa model. Ablation studies across the three categories to train MTL setup show that conversational and channel properties are important for SWDA task while disfluency relies most on conversational properties. The results justify our hypothesis that an LM that encodes the conversational, channel and ASR properties is better equipped for downstream tasks in spoken language understanding. The results also demonstrate that the properties learnt by the LM are transferable to unseen SLU tasks.

Another interesting observation is that ChatRoBERTa outperforms ASRoBERTa on SWDA dataset. Our rationale for this behavior is that SWDA dataset contains majority of utterances in grammatical forms with punctuation, which the real life dataset is devoid of. Higher resemblance of SWDA corpus to chat-dataset could be a reason for difference in performance of the two LMs.

\section{Related Works}

Recent years have seen a surge of works on the theme of neural network interpretability and understandability. A group of work has focused on unveiling secrets of language models (Clark et al., 2019; Jawahar et al., 2019). While Clark et al. (2019) probe the surface and linguistic patterns in the attention head of BERT through a set of probing tasks, Jawahar et al. (2019) throws light on structural understanding of the language captured by phrase and span representations. Other research works show limitations of LM such as ignoring negation and getting confused by simple distractors (Kassner and Schütze, 2020) in addition to BERT being inexact in encoding numeracy in its representations (Wallace et al., 2019).

There has also been research on probing LM on application specific representations such as question answering (van Aken et al., 2019), information retrieval (Yilmaz et al., 2019), recommendation systems (Penha and Hauff, 2020), dialog systems (Wu and Xiong, 2020) etc. The entire spectrum of these works aims to understand the learning capability and properties encoded in the LM along with discovering their shortcomings. The findings in these works have been utilized by the scientific community to create more robust language models: Zhang et al. (2020) propose Semantics-Aware BERT that performs better than vanilla BERT, while Wang et al. (2020b) demonstrate that incorporating sen- tence structure in pre-training LM pushes the results on downstream tasks.

However, a major section of work have primarily focused on interpreting LMs trained on clean text. SLU tasks come with their distinctive properties of ASR errors and non-grammatical, illpunctuated texts. A spoken interaction additionally requires conversational and channel understandability as highlighted in this work. While works have been carried out in understanding disfluency (Wang et al., 2020a; Lin and Wang, 2020) and turn-taking (Aldeneh et al., 2018; Hara et al., 2018), the authors narrowly aim at improving the task specific results by modelling acoustic cues (Aldeneh et al., 2018; Kumar and Vepa, 2020) or training with auxiliary tasks (Aldeneh et al., 2018; Hara et al., 2018; Wang et al., 2020a; Sundararaman et al., 2021). The effort in our work is orthogonal to what has been carried out in the past research. We investigate the representations of language model on real-life and benchmark datasets to identify the strength, limitations and possibility of a generic LM under the lens of conversational, channel and ASR properties.

\section{Conclusions and Future Work}

We investigate the representations of language model across conversational, channel and ASR properties with probing tasks such as pause, disfluency, overtalk identification, speaker role prediction, turn-tasks and ASR error type. Empirical analysis shows that LM encodes conversational and speaker-type properties to a large extent without external supervision while it has lower performance on turn-tasks and ASR error prediction. Experiments also show that a language model trained on spoken transcripts loses the linguistic understanding of dependency relations. A set of MTL experiments demonstrate the efficacy and transferability of the probe tasks to an unseen SLU dataset that advocates a need and possibility of a domain specific LM. In future, we would like to research along a few directions that originates from this work: benchmarking properties-infused LM against multimodal counterparts and revisiting the pre-training setup to identify advanced mechanisms to infuse such properties. Additionally, we would also like to evaluate on other language models such as XLNet, GPT etc. to understand the generalizability of the results. 


\section{References}

Guillaume Alain and Yoshua Bengio. 2017. Understanding intermediate layers using linear classifier probes. In 5th International Conference on Learning Representations, ICLR 2017, Toulon, France, April 24-26, 2017, Workshop Track Proceedings. OpenReview.net.

Zakaria Aldeneh, Dimitrios Dimitriadis, and Emily Mower Provost. 2018. Improving endof-turn detection in spoken dialogues by detecting speaker intentions as a secondary task. In 2018 IEEE International Conference on Acoustics, Speech and Signal Processing, ICASSP 2018, Calgary, AB, Canada, April 15-20, 2018, pages 6159-6163.

Pawel Budzianowski, Tsung-Hsien Wen, Bo-Hsiang Tseng, Iñigo Casanueva, Stefan Ultes, Osman Ramadan, and Milica Gasic. 2018. Multiwoz - A largescale multi-domain wizard-of-oz dataset for taskoriented dialogue modelling. In Proceedings of the 2018 Conference on Empirical Methods in Natural Language Processing, Brussels, Belgium, October 31 - November 4, 2018, pages 5016-5026. Association for Computational Linguistics.

Sasha Calhoun, Jean Carletta, Jason M. Brenier, Neil Mayo, Dan Jurafsky, Mark Steedman, and David Beaver. 2010. The nxt-format switchboard corpus: a rich resource for investigating the syntax, semantics, pragmatics and prosody of dialogue. Lang. Resour. Evaluation, 44(4):387-419.

Kevin Clark, Urvashi Khandelwal, Omer Levy, and Christopher D. Manning. 2019. What does BERT look at? an analysis of bert's attention. ACL Workshop BlackboxNLP: Analyzing and Interpreting Neural Networks for NLP.

Alexis Conneau, Germán Kruszewski, Guillaume Lample, Loïc Barrault, and Marco Baroni. 2018. What you can cram into a single $1 \$ \&$ !\#* vector: Probing sentence embeddings for linguistic properties. In Proceedings of the 56th Annual Meeting of the Association for Computational Linguistics, ACL 2018, Melbourne, Australia, July 15-20, 2018, Volume 1: Long Papers, pages 2126-2136. Association for Computational Linguistics.

Jacob Devlin, Ming-Wei Chang, Kenton Lee, and Kristina Toutanova. 2019. BERT: pre-training of deep bidirectional transformers for language understanding. In Proceedings of the 2019 Conference of the North American Chapter of the Association for Computational Linguistics: Human Language Technologies, NAACL-HLT 2019, MN, USA, June 2-7, 2019, pages 4171-4186.

Tanvi Dinkar, Ioana Vasilescu, Catherine Pelachaud, and Chloé Clavel. 2020. How confident are you? exploring the role of fillers in the automatic prediction of a speaker's confidence. In 2020 IEEE International Conference on Acoustics, Speech and Signal
Processing, ICASSP 2020, Barcelona, Spain, May 4-8, 2020, pages 8104-8108.

Efthymios Georgiou, Charilaos Papaioannou, and Alexandros Potamianos. 2019. Deep hierarchical fusion with application in sentiment analysis. In Interspeech 2019, 20th Annual Conference of the International Speech Communication Association, Graz, Austria, 15-19 September 2019, pages 1646-1650.

Kohei Hara, Koji Inoue, Katsuya Takanashi, and Tatsuya Kawahara. 2018. Prediction of turn-taking using multitask learning with prediction of backchannels and fillers. In Interspeech 2018, 19th Annual Conference of the International Speech Communication Association, Hyderabad, India, 2-6 September 2018, pages 991-995.

Ganesh Jawahar, Benoît Sagot, and Djamé Seddah. 2019. What does BERT learn about the structure of language? In Proceedings of the 57th Conference of the Association for Computational Linguistics, ACL 2019, Florence, Italy, pages 3651-3657.

Nora Kassner and Hinrich Schütze. 2020. Negated and misprimed probes for pretrained language models: Birds can talk, but cannot fly. In Proceedings of the 58th Annual Meeting of the Association for Computational Linguistics, ACL, pages 7811-7818.

Jonggu Kim, Yewon Jeong, and Jong-Hyeok Lee. 2020. Speaker-informed time-and-content-aware attention for spoken language understanding. Computer Speech \& Language, 60:101022.

Ayush Kumar and Jithendra Vepa. 2020. Gated mechanism for attention based multi modal sentiment analysis. In 2020 IEEE International Conference on Acoustics, Speech and Signal Processing, ICASSP 2020, Barcelona, Spain, pages 4477-4481.

Zhongli Li, Qingyu Zhou, Chao Li, Ke Xu, and Yunbo Cao. 2020. Improving BERT with syntax-aware local attention. CoRR, abs/2012.15150.

Binghuai Lin and Liyuan Wang. 2020. Joint prediction of punctuation and disfluency in speech transcripts. In Interspeech 2020, 21st Annual Conference of the International Speech Communication Association, Virtual Event, Shanghai, China, pages 716-720.

Yinhan Liu, Myle Ott, Naman Goyal, Jingfei Du, Mandar Joshi, Danqi Chen, Omer Levy, Mike Lewis, Luke Zettlemoyer, and Veselin Stoyanov. 2019. Roberta: A robustly optimized BERT pretraining approach. CoRR, abs/1907.11692.

Mitchell P. Marcus, Beatrice Santorini, and Mary Ann Marcinkiewicz. 1993. Building a large annotated corpus of english: The penn treebank. Comput. Linguistics, 19(2):313-330.

Mahdi Namazifar, Gökhan Tür, and Dilek Hakkani-Tür. 2021. Warped language models for noise robust language understanding. In IEEE Spoken Language 
Technology Workshop, SLT 2021, Shenzhen, China, January 19-22, 2021, pages 981-988. IEEE.

Vassil Panayotov, Guoguo Chen, Daniel Povey, and Sanjeev Khudanpur. 2015. Librispeech: An ASR corpus based on public domain audio books. In 2015 IEEE International Conference on Acoustics, Speech and Signal Processing, ICASSP 2015, South Brisbane, Queensland, Australia, April 19-24, 2015, pages 5206-5210. IEEE.

Gustavo Penha and Claudia Hauff. 2020. What does BERT know about books, movies and music? probing BERT for conversational recommendation. In RecSys 2020: Fourteenth ACM Conference on Recommender Systems, Virtual Event, Brazil, September 22-26, 2020, pages 388-397.

Matthew Roddy, Gabriel Skantze, and Naomi Harte. 2018. Investigating speech features for continuous turn-taking prediction using lstms. In Interspeech 2018, 19th Annual Conference of the International Speech Communication Association, Hyderabad, India, 2-6 September 2018, pages 586-590.

Dhanasekar Sundararaman, Vivek Subramanian, Guoyin Wang, Shijing Si, Dinghan Shen, Dong Wang, and Lawrence Carin. 2019. Syntax-infused transformer and BERT models for machine translation and natural language understanding. CoRR, abs/1911.06156.

Mukuntha Narayanan Sundararaman, Ayush Kumar, and Jithendra Vepa. 2021. PhonemeBERT: Joint Language Modelling of Phoneme Sequence and ASR Transcript. In Proc. Interspeech 2021, pages 3236-3240.

Ian Tenney, Patrick Xia, Berlin Chen, Alex Wang, Adam Poliak, R. Thomas McCoy, Najoung Kim, Benjamin Van Durme, Samuel R. Bowman, Dipanjan Das, and Ellie Pavlick. 2019. What do you learn from context? probing for sentence structure in contextualized word representations. In 7th International Conference on Learning Representations, ICLR 2019, New Orleans, LA, USA.

Betty van Aken, Benjamin Winter, Alexander Löser, and Felix A. Gers. 2019. How does BERT answer questions?: A layer-wise analysis of transformer representations. In Proceedings of the 28th ACM International Conference on Information and Knowledge Management, CIKM 2019, Beijing, China, pages 1823-1832.

Eric Wallace, Yizhong Wang, Sujian Li, Sameer Singh, and Matt Gardner. 2019. Do NLP models know numbers? probing numeracy in embeddings. In Proceedings of the 2019 Conference on Empirical Methods in Natural Language Processing and the 9th International Joint Conference on Natural Language Processing, EMNLP-IJCNLP 2019, Hong Kong, China, pages 5306-5314.
Shaolei Wang, Wanxiang Che, Qi Liu, Pengda Qin, Ting Liu, and William Yang Wang. 2020a. Multitask self-supervised learning for disfluency detection. In The Thirty-Fourth AAAI Conference on Artificial Intelligence, AAAI 2020, The Thirty-Second Innovative Applications of Artificial Intelligence Conference, IAAI 2020, The Tenth AAAI Symposium on Educational Advances in Artificial Intelligence, EAAI 2020, New York, NY, USA, February 7-12, 2020, pages 9193-9200.

Wei Wang, Bin Bi, Ming Yan, Chen Wu, Jiangnan Xia, Zuyi Bao, Liwei Peng, and Luo Si. 2020b. Structbert: Incorporating language structures into pre-training for deep language understanding. In 8th International Conference on Learning Representations, ICLR 2020, Addis Ababa, Ethiopia.

Jochen Weiner, Mathis Engelbart, and Tanja Schultz. 2017. Manual and automatic transcriptions in dementia detection from speech. In Interspeech 2017, 18th Annual Conference of the International Speech Communication Association, Stockholm, Sweden, August 20-24, 2017, pages 3117-3121.

Chien-Sheng Wu and Caiming Xiong. 2020. Probing task-oriented dialogue representation from language models. In Proceedings of the 2020 Conference on Empirical Methods in Natural Language Processing, EMNLP 2020, Online, November 16-20, 2020, pages 5036-5051.

Zeynep Akkalyoncu Yilmaz, Wei Yang, Haotian Zhang, and Jimmy Lin. 2019. Cross-domain modeling of sentence-level evidence for document retrieval. In Proceedings of the 2019 Conference on Empirical Methods in Natural Language Processing and the 9th International Joint Conference on Natural Language Processing, EMNLP-IJCNLP 2019, Hong Kong, China, November 3-7, 2019, pages 3488-3494.

Vicky Zayats, Trang Tran, Richard A. Wright, Courtney Mansfield, and Mari Ostendorf. 2019. Disfluencies and human speech transcription errors. In Interspeech 2019, 20th Annual Conference of the International Speech Communication Association, Graz, Austria, 15-19 September 2019, pages 3088-3092.

Rui Zhang, Honglak Lee, Lazaros Polymenakos, and Dragomir R. Radev. 2018a. Addressee and response selection in multi-party conversations with speaker interaction rnns. In Proceedings of the ThirtySecond AAAI Conference on Artificial Intelligence, (AAAI-18), the 30th innovative Applications of Artificial Intelligence (IAAI-18), and the 8th AAAI Symposium on Educational Advances in Artificial Intelligence (EAAI-18), New Orleans, Louisiana, USA, February 2-7, 2018, pages 5690-5697.

Saizheng Zhang, Emily Dinan, Jack Urbanek, Arthur Szlam, Douwe Kiela, and Jason Weston. 2018b. Personalizing dialogue agents: I have a dog, do you have pets too? In Proceedings of the 56th Annual 
Meeting of the Association for Computational Linguistics, ACL 2018, Melbourne, Australia, July 1520, 2018, Volume 1: Long Papers, pages 2204-2213. Association for Computational Linguistics.

Zhuosheng Zhang, Yuwei Wu, Hai Zhao, Zuchao Li, Shuailiang Zhang, Xi Zhou, and Xiang Zhou. 2020. Semantics-aware BERT for language understanding. In The Thirty-Fourth AAAI Conference on Artificial Intelligence, AAAI 2020, The Thirty-Second Innovative Applications of Artificial Intelligence Conference, IAAI 2020, 10th AAAI Symposium on Educational Advances in Artificial Intelligence, EAAI 2020, New York, NY, USA, February 7-12, 2020, pages 9628-9635.

\section{A Appendix}

\section{A.1 Dataset Creation}

In this section, we describe the methodology used to curate the dataset for each probing tasks mentioned in Section 2.

\section{A.1.1 Conversational Properties}

- Disfluency Detection: To create the dataset for disfluency we follow two-step process: i) candidate retrieval, ii) manual annotation. For candidate retrieval, we have a couple of pipelines, one relying on lexical cues and another taking hints from the speech cues. For lexical cues, we use a simple keyphrase based lookup to identify the candidate speaker utterances containing markers for disfluency such as repetition of words, filler words and discourse markers. We, however, note that lexical cues only forms a subset of all disfluencies. To include speech induced disfluencies, we identify turns that contains multiple intermittent pauses within a short time frame. This candidate extraction ensures that we are probing a lexical model on its capability to understand disfluencies that are not keyword based and hence a greater semantic understanding is needed to locate such disfluent contexts in the spoken transcripts. Once such candidates are obtained, we use manual annotators to label them into fluent and disfluent categories, thus generating a binary classification dataset.

- Pause Identification: The dataset for pause identification is generated by retrieving all utterances where speaker takes one or more pauses longer than 5 seconds in an individual turn. Additionally, we exclude all such utterances that could be a disfluent candidate. The rationale behind such decision is to ensure that we not add bias in the pause identification probe setup such that disfluent markers lead to a better pause understanding. In this case, there is no need for manual annotation as the pause duration obtained from the speech information acts as gold label for the utterances. We frame pause identification as a binary classification where one class denotes that speaker takes a pause longer than $5 \mathrm{sec}$. in a given turn of the conversation while other class denotes the absence of pauses in the turn.

- Overtalk Detection: An overtalk in a given dual speaker conversation would like Table 6. These leads to broken and incomprehensible turns compared to their clean versions of transcripts. While it is easy to detect such overtalk when the recording lines itself are unique to speakers, this is an arduous task for monochannel recordings. Thus, we curiously probe the language model to see if it is able to detect transcripts with overtalks. We follow a simple mechanism to curate overtalk dataset: we identify overtalk via rule based system from dual channel call and create corresponding monochannel transcripts (AB-mono in $\mathrm{Ta}-$ ble 6). These monochannel transcripts are dataset that belong to 'overtalk' label. Any non-overtalk transcript from a single speaker belong to 'non-overtalk' label. Thus, overtalk detection too is a binary classification task.

\begin{tabular}{ll} 
Speaker & Transcript \\
A & i'm \\
B & yes \\
A & not \\
B & i know \\
A & referring to \\
B & how \\
A & transaction \\
B & it works \\
\hline A - clean & i'm not referring to transaction \\
B - clean & yes $i$ know how it works \\
\hline AB-mono & i'm yes not $i$ know referring to how \\
& transaction it works
\end{tabular}

Table 6: An example of a dual channel transcript containing overtalk. A-clean, B-clean refers to rewriting what A and B's transcript would look like if had they not spoken over each other.

- Question Identification: Question identification is a non-trivial problem in spoken language transcripts due to inaccurate predictions and non-surface question constructs, such as: 
and you would like me to continue. In the example, the speaker intends to actually ask a question if he/she can continue further. To create question dataset, we follow a similar two step process: i) candidate generation; and ii) manual annotation. For candidate generation, we two approaches: a) question keyphrases based lookup; and b) using speaker replies that indicate presence of boolen answers such as yes that's true or entities such as sure, it would be ninety five dollars. Once candidate turns are collected, we ask manual annotators to mark the question span and the category of the question.

\section{A.1.2 Channel Properties}

- Speaker Role: We restrict the domain of the dataset to call center conversations with two speaker roles: a) agent and b) customer. These two speakers have distinctive properties as per their role, agent are expected to be fluent while being empathetic and enthusiastic towards the customers. Customers, on the other hand, are calling to raise their concerns or ask for help with many cases of heated engagements with agents. Thus, we create a speaker role binary classification dataset with two classes: agent and customer obtained directly from dual channel calls.

- Response-Length: To create dataset for this task, we use the time-duration of an utterance's response to categorize it into a short response if it is is less than 30 seconds of duration. For response lasting longer than 30 seconds are considered to be long response. The utterance is what is taken as consideration in the dataset with the label being short or long depending on the utterance's response length. To arrive at the duration, we discard turns in the initial 5\%ile and last 5\% ile category based on the turn duration in order to remove outliers. For the remaining dataset, we choose the time period that belongs to the 50th percentile.

- Turn-Taking: We pose the task as a binary classification task where a label of 'turncontinue' would mean that with the given utterance, the speaker has not yet finished speaking and hence would add on further, while a label of 'turn-break' would refer to the sce- nario where speaker has finished its turn of speaking.

\section{A.1.3 ASR Properties}

- Error Type Prediction: We obtain spoken transcripts from automatic speech recognition system. Additionally, we get manual transcripts for the same set of calls. We run alignment over the parallel corpus to identify the categories of errors for each token in spoken transcripts. We formulate two tasks for error type prediction: binary classification where the model only needs to predict if the token under consideration is erroneous; and multiclass classification in which for any erroneous token, the model needs to predict the error type: insertion, deletion or substitution.

- WER Prediction: From the dataset obtained in the above task, we compute the word error rate for the spoken transcript as compared to manual transcripts. A regression model is trained for this task.

\section{A.2 Token Baseline}

In addition to the three setups present in Section 5, we also evaluate a ngram based token baseline to contrast the performance observed from language model based probe classifiers. We use ngram features upto quadgrams as input to the model. We use logistic and linear regression for all classification and regression tasks respectively. The results for token baseline is presented in Table 7 .

\begin{tabular}{l|c} 
Probe Tasks & Token Baseline \\
\hline Pause & 70.05 \\
Disfluency & 66.55 \\
Overtalk & 60.94 \\
Question & 69.49 \\
\hline Speaker & 79.75 \\
Response-Length & 65.25 \\
Turn-Taking & 58.29 \\
\hline Binary & 54.34 \\
Insertion & 50.29 \\
Deletion & 49.26 \\
Substitution & 41.33 \\
WER & 10.31
\end{tabular}

Table 7

\section{A.3 Attention Scores}

In this section, we show the variation in average attention scores between a pair of tokens across 


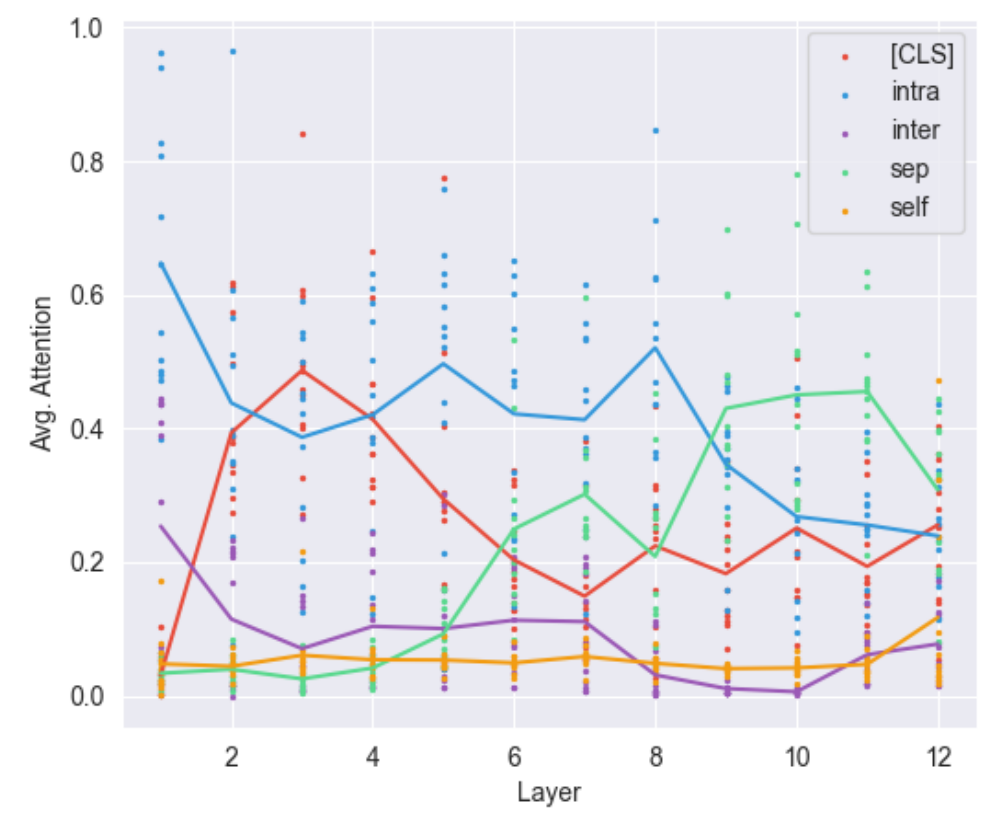

(a) Average attention scores for ASRoBERTa across all layers. The graph shows that inter-sentence attention (ISA) is low for all layers except the initial layer. [CLS] and sep corresponds to $<s>$ and $</ s>$ in ASRoBERTa.

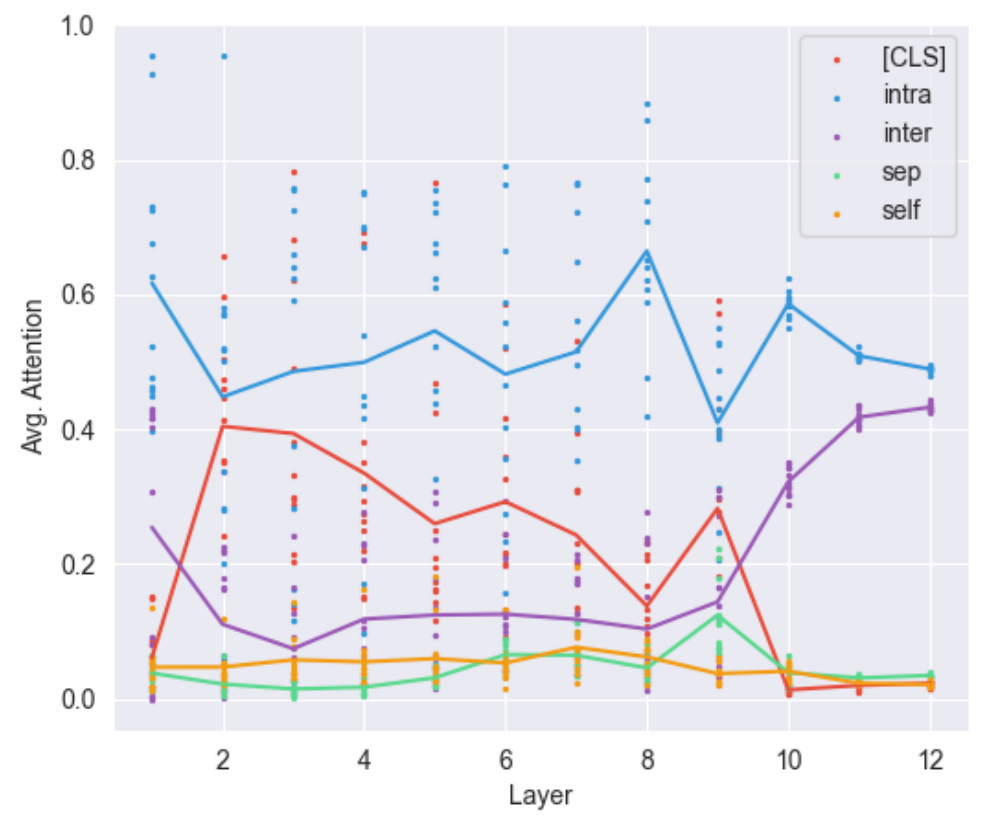

(b) Average attention scores across all layers for ASRoBERTa trained on next-sentenceprediction (NSP) task. The graph shows that inter-sentence attention (ISA) goes up, especially for final layer suggesting that inter-sentence understanding is needed for solving NSP task. [CLS] and sep corresponds to $<s>$ and $</ s>$ in ASRoBERTa. 
all layers for ASRoBERTa model trained only on masked language modelling (MLM) task and ASRoBERTa-NSP model which is further finetuned on next-sentence-prediction (NSP) task. The graphs demonstrate that inter-sentence scores rise in final layers (Figure 1b) indicating the need of inter-sentence interaction for solving NSP task. The code for graph generation has been adapted from the paper by Clark et al. (2019). 